\title{
2012 ACCF/AATS/SCAI/STS expert consensus document on transcatheter aortic valve replacement: Executive summary
}

\author{
Arvind Agnihotri, MD
}

\section{INTRODUCTION}

The American Association for Thoracic Surgery (AATS), along with 11 other professional societies, participated in the writing and approval of a consensus document on transcatheter aortic valve replacement (TAVR), which was recently published in the Journal of the American College of Cardiology in March 2012 (and also published online in its entirety on the JTCVS and AATS Web sites). The 55-page document provides a review of the current status of TAVR, including patient evaluation, decision making regarding treatment options, implantation details, postoperative considerations, and future directions. The work serves as a reference for practitioners directly involved in TAVR, and also as a resource for the larger group of physicians seeking to understand this technology and its role in the treatment of aortic stenosis (AS).

\section{AORTIC STENOSIS}

With the onset of symptoms, the natural history of AS is poor. Medically managed inoperable patients in the Placement of Aortic Transcatheter Valve (PARTNER) trial had a 50\% 1-year mortality rate, yet there are many patients who are not treated because of elevated risk. Diagnosis is generally made by echocardiography, with severe stenosis defined as a peak velocity of greater than $4 \mathrm{~m} / \mathrm{s}$ OR a valve area of $<1 \mathrm{~cm}^{2}$ when systolic function is normal (indexed $<0.6 \mathrm{~cm}^{2} / \mathrm{m}^{2}$ ). Diagnostic challenges exist in the presence of significant aortic regurgitation, upper septal hypertrophy, and in the failing ventricle. Patients with low gradient AS most likely to benefit from treatment are those with contractile reserve (as shown by dobutamine infusion), limited coronary disease, and a mean gradient of $\geq 20 \mathrm{~mm} \mathrm{Hg}$.

\section{OPEN SURGERY}

Open surgical replacement (AVR) remains the only effective treatment considered a class I recommendation by the ACCF/AHA guidelines in adults with severe symptomatic AS. Current data from the Society of Thoracic Suergons (STS) Registry documents a mortality rate that is $2.6 \%$ for

\footnotetext{
From Cardiac Surgery, Department of Surgery, Massachusetts General Hospital, Boston, Mass; and Harvard Medical School, Boston, Mass.

Disclosures: Author has nothing to disclose with regard to commercial support.

Address for reprints: Arvind Agnihotri, MD, Cardiac Surgery, Massachusetts General Hospital, COX 642, 55 Fruit Street, Boston, MA 02114 (E-mail: aagnihotri@ partners.org).

J Thorac Cardiovasc Surg 2012;144:534-7

$0022-5223 / \$ 36.00$

Copyright (c) 2012 by The American Association for Thoracic Surgery

http://dx.doi.org/10.1016/j.jtcvs.2012.07.021
}

all patients undergoing AVR, and in patients with minimal comorbidity, mortality and major morbidity rates are under $1 \%$ each in many centers. Surgical AVR for most patients is a proven low-risk therapy for AS and remains the gold standard.

\section{PATIENT SELECTION}

Indications for treatment in AS are well established by guidelines; however, decisions at the extreme of risk can be complex and is facilitated by use of risk models. The STS and logistic EuroSCORE are the most commonly used mechanisms for mathematical estimation of surgical risk. Along with consideration of the patient's nonrepresented risk (risk not accounted for in the models), the patient might be declared "prohibitive risk," "extreme risk," or "inoperable," but it is acknowledged that these terms are used with some subjectivity.

The alternatives to surgical AVR are limited to medical therapy and percutaneous balloon valuloplasty. Percutaneous balloon valuloplasty is known to often result in hemodynamic improvement, but this does not translate in to survival benefit and, as such, its role is strictly palliative or, in rare circumstances, as a bridge toward more definitive therapy.

\section{TAVR BACKGROUND}

In the 1990s, investigators began evaluation of stentbased porcine bioprosthesis delivered to various aortic sites in animal models. In 2000, a percutaneous heart valve was placed in a child's pulmonary arterial conduit, and in 2002, the first human TAVR was performed. Since then, multiple devices have been evaluated, but most human data comes from 2 valve designs: The Sapien valve (Edwards Lifesciences, Inc, Irvine, Calif) and the CoreValve (Medtronic, Inc, Minneapolis, Minn). The Sapien is available in 23- and 26-mm sizes; the CoreValve in 23, 26, and $29 \mathrm{~mm}$. The Sapien valve is mounted within a balloon-expandable cobalt chromium stent, whereas the CoreValve is self-expanding with a nitinol frame. Initial delivery systems required 22or 24-French sheaths, but recent iterations have decreased this to 18 French. Both valves are preferentially placed via a transfermoral arteral (TF) approach. The Sapien valve is also implantable via a transapical (TA) route, using a specialized delivery system (Ascendra; Edwards Lifesciences).

\section{CLINICAL EVIDENCE: REGISTRIES}

Initial evidence regarding the outcomes of TAVR with Sapien and CoreValve come from registry data. The patients 


$$
\begin{aligned}
& \text { Abbreviations and Acronyms } \\
& \text { AS = aortic stenosis } \\
& \text { AVR }=\text { aortic valve replacement } \\
& \text { LV } \quad=\text { left ventricular } \\
& \text { PARTNER }=\text { Placement of Aortic Transcatheter } \\
& \text { Valve trial } \\
& \text { STS }=\text { Society of Thoracic Surgeons } \\
& \text { TA }=\text { transapical } \\
& \text { TAVR }=\text { transcatheter aortic valve } \\
& \text { replacement } \\
& \mathrm{TF} \quad=\text { transfermoral arteral }
\end{aligned}
$$

enrolled in the multiple European registries comprised a high-risk group (generally age $>80$ years and EuroSCORE $>20$ ), although there was variability and resultant difficultly in determining whether the included patients were truly inoperable or simply at elevated risk. Regardless, the data showed acceptable mortality and morbidity for both valves-30-day mortality rate ranged from $8 \%$ to $10 \%$ in most centers. Early stroke occurred at a rate of $2.5 \%$ to $5 \%$. With both valves, patients undergoing successful TAVR enjoyed symptom relief similar to that seen with surgical AVR. Immediate postprocedural gradients in TAVR valves appeared to be about $10 \%$ lower (superior) than surgical prosthetics. There is limited information regarding durability beyond 5 years in registry data, but valve failure was not observed.

\section{EVIDENCE: PARTNER TRIAL}

The PARTNER trial was a prospective, unblinded, randomized, multicenter trial evaluating the safety and effectiveness of the Sapien valve. Two populations were enrolled: High-risk (cohort A; expected risk of death at 30 days, $>15 \%$ ), and inoperable (cohort B). Inoperability was formally defined as " $>50 \%$ predicted probability of mortality or serious irreversible complication by 30 days 1 cardiologist and 2 surgeons." Randomization was stratified based on suitability of vascular access for TF delivery. Cohort A patients who were TF candidates were randomized to TAVR versus surgical AVR. Cohort A patients who were not eligible for TF were randomized to TA TAVR versus surgical AVR. Inoperable patients were randomized to TF TAVR versus medical therapy. Patients who were considered inoperable but who did not meet criteria for TF delivery were not enrolled. Twenty-five sites participated, enrolling 699 patients in cohort A and 358 patients in cohort B.

In the inoperable patients (cohort B), TAVR was found to substantially reduce all-cause mortality by nearly $50 \%$ at 1-year follow-up. In addition, all key secondary end points, including patient function, significantly improved at 30 days and 1 year. TAVR was associated with an increased risk of stroke and procedure-related adverse events such as bleeding and vascular complications, but the benefit appears to greatly exceed the risks.

In the high-risk (cohort A) patients, TAVR was noninferior to AVR for all-cause mortality at 1 year $(24.2 \%$ vs $26.8 \%$ ). Some morbidity was more frequent with TAVR such as stroke (3.8\% vs $2.1 \%$ ) and vascular complications $(11.1 \%$ vs $3.2 \%)$. In contrast, major bleeding and atrial fibrillation were more common with surgical AVR. The data support TAVR as an acceptable alternative to surgical AVR in selected high-risk but operable patients.

\section{PATIENT EVALUATION FOR TAVR}

One of the lessons of TAVR experience has been the importance of a multidisciplinary approach to the management of patients with AS. Localization of a heart team working together in a valve clinic will optimize communication and facilitate rapid evaluation.

In the US, only the Sapien valve is approved for use via a TF route. Indications for clinical application should be based on inclusion criteria used for the PARTNER trial cohort B (inoperable). Patients should have symptomatic AS (New York Heart Association class 2 or greater), and documented severe AS (mean gradient $>40 \mathrm{~mm} \mathrm{Hg}$ or jet velocity $>4.0 \mathrm{~m} / \mathrm{s}$ AND valve area $<0 / 8 \mathrm{~cm}^{2}$ or indexed area $<0.5 \mathrm{~cm}^{2} / \mathrm{m}^{2}$ ). In addition, a cardiac interventionalist and 2 surgeons must agree that the patient is too high a risk for surgical AVR.

Specific exclusion criteria must be noted, in particular native annular size $<18 \mathrm{~mm}$ or $>25 \mathrm{~mm}$, bicuspid aortic valve, severe mitral regurgitation, left ventricular (LV) ejection fraction $<20 \%$, renal failure, and estimated life expectancy $<12$ months. Complex decisions regarding appropriateness (futility) of treatment in the very frail or those with multiple comorbidities require team discussion and consensus.

When the decision is made to consider TAVR, assessment screening should include echocardiography and tomographic imaging. Echocardiography (transthoracic echocardiography or transesophageal echocardiography) must yield a reliable measurement of annular size, generally measured anteroposteriorly in a long-axis view. Care must be taken to measure the true annulus and not the overlying calcium. Overstimation of annular size can result in placement of a valve that is too large with the increased risk of incomplete expansion of the construct or catastrophic annular rupture. Underestimation can lead to increased paravalular leakage or unstable valve deployment. Additional considerations include confirmation of a tri-leaflet valve, presence and severity of aortic insufficiency, and relation of the coronary arteries.

Tomographic imaging is also critical for evaluation of the size and quality of the vascular tree. In addition to determination of adequacy of vascular dimensions, imaging provides information on other important vascular pathology such as the presence and location of calicification, degree of 
tortuosity, and presence of thrombus. Multi-detector computed tomography with electrocardiogram synchronization can supplement information obtained by echocardiography and give a comprehensive picture of root anatomy.

\section{PROCEDURAL PERFORMANCE}

The joint conduct of the procedure by the surgeon and cardiologist is important for procedural success in TAVR. The procedure is performed in a hybrid operating environment, either located in the catherization laboratory suitable converted or in a traditional operating room equipped with imaging capabilities. Optimal equipment includes a state-of-the-art, large field-of-view floroscopic imaging system-fixed overhead or floor mounted. A portable C-arm is not sufficient. There should be availability of transesophageal echocardiography, general anesthesia services, equipment for coronary intervention and open surgery, and the ability to place the patient on cardioplumonary bypass, although the latter is needed in less than $5 \%$ of cases.

TAVR is typically performed under general anesthesia with monitoring of arterial pressure, pulmonary artery pressure, and transesophageal echocardiography. A temporary pacing wire is either placed in the right ventricle from via the central catheter or from a femoral venous route. Hypothermia is avoided in a similar manner to patients undergoing off-pump coronary artery bypass grafting. Patients should be well hydrated, and inotropes are instituted when the cardiac index is less than $1.8 \mathrm{~L} / \mathrm{min} / \mathrm{m}^{2}$. The mean pressure should be maintained at $>75 \mathrm{~mm} \mathrm{Hg}$ before rapid pacing to avoid the cycle of hypotension and subendocardial ischemia than can rapidly lead to hemodynamic collapse and fibrillation. Anticoagulation is used with a target activated clotting time of $>300$ seconds. Heparin may be reversed at the completion of the procedure, although this may be unnecessary with an uneventful percutaneous femoral approach.

Both a percutaneous and cutdown approach can be used for TF-TAVR, the former used in experienced centers for only favorable quality femoral vessels. Once access is obtained, a series of dilators is used under fluoroscopic guidance, with careful attention to the resistance required. The sheath is then passed into the body of the thoracoabdominal aorta. Standard techniques are used to cross the aortic valve retrograde, and a stiff wire exchange performed.

Recently, retrograde access has also been advocated via a direct ascending aortic technique, with exposure obtained via either an upper partial sternotomy or a small right thoracotomy. There is limited but encouraging experience with auxiliary artery access as another alternative retrograde approach.

The TA approach is the only currently available antegrade approach and specialized equipment is available only for the Sapien valve. Access is obtained via a small left thorocotomy, with a site chosen based on fluroscopic and/or echocardiographic guidance. Removal of a small segment of rib may avoid rib fractures and resulting pain. Soft tissue retractors are preferred over metal retractors for similar reasons. The pericardium is opened and the LV apex is identified. Either purse string or mattress sutures are placed, and a direct puncture allows passage of a guide wire into the LV and across the aortic valve, with care taken to avoid the mitral apparatus. Guiding catheters allow positioning in the thoracic aorta and a wire exchange is performed for a stiff wire.

After a balloon valuloplasy, the replacement valve is moved into position. Occasionally, retrograde crossing of the heavily calcified valve can be difficult may require additional steps such as placement of a small balloon to prop open the native valve (buddy balloon technique). The appropriate camera angle for viewing the aortic annulus "in plane" is determined by root angiography. Final precise positioning is accomplished with a combination of fluroscopy and echocardiography. Deployment then takes place followed immediately by echocardiographic evaluation.

\section{COMPLICATIONS}

Stoke incidence with TAVR is of concern. In the PARTNER cohort A, the risk of clinically apparent "major" stroke (Rankin score $\geq 2$ ) was $3.8 \%$ at 30 days and $5.1 \%$ at 1 year among the TAVR group compared with $2.1 \%$ and $2.4 \%$, respectively, in the surgical group. In cohort B, the stroke risk was $5 \%$ with TAVR versus $1.1 \%$ with standard therapy at 30 days and $8.4 \%$ versus $3.9 \%$ at 1 year.

TAVR may cause conduction abnormalities through mechanical impingement of the conduction system by the prosthesis or displaced calcific valve. The incidence of complete heart block requiring pacemaker implantation has been higher with the CoreValve $(19.2 \%-42.5 \%)$ than the Sapien Valve (1.8\%-8.5\%), potentially due to its larger profile, nitinol construction, and extension in the left ventricular outflow tract. In contrast, surgical AVR pacemaker rates are generally less than $10 \%$.

Vascular complications are the most frequent adverse event with TF-TAVR, with major events occurring at a rate of $2 \%$ to $26 \%$. Predictors include the operator experience, presence and location of vascular calcification, vascular tortuosity, and sheath-to-artery ratio.

Hemodynamic collapse during the procedure or immediately after deployment can occur, often triggered by the induced hypotension of rapid pacing. Specific measures must be taken to mitigate this risk, including adequate volume loading, minimization of rapid pacing duration, and proactive anesthetic management of blood pressure. In patients felt to be at elevated risk of collapse, cardioplumonary bypass may be used electively. Low-output states are common both pre- and postdeployment and aggressively therapy is 
indicated, including the use of inotropes and the intra-aortic balloon pump.

Rarely, TAVR devices will be malpositioned and may even emoblize. In the self-expanding device (CoreValve), the construct can be moved to some degree until fully deployed, but with the ballon-expandable device (Sapien), there is only 1 opportunity to obtain the correct position. Malpositioned valves may not function adequately (severe aortic insufficiency) or be unstable. When unstable, a second valve ("valve-in-valve") may capture the first construct and secure both to the annulus. Embolization has occurred, both in the LV and into the aorta. The former uniformly requires urgent surgery and the later can often be managed with deployment into the thoracic aorta.

Postdeployment aortic regurgitation is usually paravalular and is common ( $85 \%$ of patients). Repeat ballooning may reduce leaks and is routinely used when they are large. At 1 year, up to $75 \%$ of patients after TAVR still have mild or more paravalular regurgitation. Hemolysis has not been reported but the clinical significance of paravalular leakage is not well characterized.

Additional complications are rare and include annular rupture, coronary occlusion, and ventricular perforation. With the TA approach, LV disruption can occur, necessitating the use of cardioplumary bypass to facilitate attempted repair.

\section{FUTURE DIRECTIONS}

Next-generation devices are in various stages of development and testing and promise the potential for improvements-lower profile delivery systems, more accurate positioning, reduced paravalular leakage, and the ability to reposition or retrieve. Many patients with dysfunction of a conventional aortic prosthesis can be treated by TAVR, placing the new valve within the old prosthesis (valve-in-valve), and represent a population of active investigation.
Based on PARTNER results, TAVR clearly has a role in the management of the inoperable patient and it appears to be appropriate in the very high-risk patient (STS mortality estimate $>8 \%$ ), but the proper application in the intermediate risk (STS >4) operable patient is not yet known and is currently under investigation (PARTNER II trial).

\section{EUROPEAN EXPERIENCE}

The European and American experiences with TAVR have been in stark contrast because of the regulatory differences that, until recently, limited use in the US to centers participating in national trials. In some centers, TAVR now accounts for over $30 \%$ of AVRs. The models developed in Europe for training of TAVR centers are being replicated in the US, and include didactic sessions, simulator training, observation of cases at experience centers, and proctoring at new centers. Monitoring of European outcomes and usage is facilitated by use of registries and a similar system is now in place in the US.

\section{SUMMARY AND RECOMMENDATIONS}

TAVR will undoubtedly become an increasing widespread and important treatment option for patients with AS. The recent approval of TAVR in the US allows for expansion of centers for commercial implantation. Several recommendations can be made based on the available evidence and current experience. This is a complex procedure that requires meticulous attention to detail to avoid complications, and the multi-specialty nature requires a team-based approach at selected high-volume centers. Patient selection should be based on published randomized data: TAVR is recommended in patients with prohibitive risk who are judged to have the potential to benefit, and it is a reasonable option in operable patients at very high risk. Indications are likely to evolve and encompass additional patients. Every center should enroll patients in a registry. 